\title{
CD133-positive Status Predicts Better Prognosis in Metastatic Colorectal Cancer Patients Treated with Cetuximab
}

\section{Dai Inoue $^{1}$, Satoshi Matsusaka ${ }^{1,3 *}$, Noiriko Yamamoto ${ }^{2}$, Mitsukuni Suenaga $^{1}$, Eiji Shinozaki ${ }^{1}$, Nobuyuki Mizunuma ${ }^{1}$, Yuichi Ishikawa ${ }^{2}$ and Kiyohiko Hatake ${ }^{3}$}

${ }^{1}$ Department of Gastroenterology, Cancer Institute Hospital, Japan

${ }^{2}$ Department of Pathology, Cancer Institute Hospital, Japan

${ }^{3}$ Clinical Chemotherapy, Cancer Chemotherapy Center of Japanese Foundation for Cancer Research, Japan

\begin{abstract}
Background: There are few evidence which kind of tumor is more benefit from cetuximab, more investigations of supplementary predictive factors are highly relevant. The purpose is to evaluate the prognostic significance of CD133 expression and to investigate the utility as biomarker of prognosis of metastatic colorectal cancer (mCRC) patients treated with cetuximab.

Methods: We investigated progression-free survival (PFS) and overall survival (OS) of patients with mCRC about 5FU-based regimen as first line chemotherapy and cetuximab as salvage line retrospectively, and performed immunohistochemical staining to evaluate the CD133 status. Non-parametric statistics, univariate and multivariate analysis were used.

Results: From October 2008 to June 2009, 46 patients with measurable mCRC had received cetuximab as salvage line, and all of them had treated with 5FU-based regimen as first line. In first line chemotherapy, the median OS was 33.1 months $(95 \% \mathrm{Cl}, 25.6-40.4)$ in the CD133-positive patients as compared with 16.0 months $(95 \% \mathrm{Cl}$, 0.938-31.1) in the CD133-negative patients $(P=0.004)$, but there were no statistically significant difference in PFS between both group. In salvage therapy with cetuximab, the median OS was 8.8 months $(95 \% \mathrm{Cl}, 5.24-12.4)$ in the CD133-positive patients as compared with 5.1 months $(95 \% \mathrm{Cl}, 3.23-6.90)$ in the CD133-negative patients $(P=0.007)$. The median PFS was also significantly better in CD133-positive patients, with a median of 5.2 month $(95 \% \mathrm{Cl}, 2.96-7.51)$ in the CD133-positive patients as compared with 2.8 month (95\% Cl, 1.48-4.06) in CD133negative patients $(P=0.046)$. Univariate and multivariate Cox proportional hazards regression was performed, and it is shown that CD133 predicted OS and PFS of cetuximab.
\end{abstract}

Conclusions: CD133 status was correlated with the prognosis of patients treated with cetuximab, suggesting that $C D 133$ can be a useful biomarker of $\mathrm{mCRC}$ treated with cetuximab.

Keywords: CD133; Cetuximab; EGFR; Colorectal cancer; Immunohistochemistry

\section{Introduction}

Significant improvements have been achieved in the treatment of metastatic colorectal cancer (mCRC) with the development of new regimens such as FOLFOX and FOLFIRI. Some of these new therapeutic agents are molecularly-targeted such as those against monoclonal antibody against epidermal growth factor receptor (EGFR) or vascular endothelial growth factor. Cetuximab, an IgG1 anti-EGFR chimeric mouse/human monoclonal antibody, induces synergistic antitumor activity when combined with chemotherapy [1-5]. Selection of patients for EGFR-targeted monoclonal antibodies based on tumor KRAS analysis is a major step towards tailored treatment in mCRC. Previous studies have shown that response to anti-EGFR monoclonal antibodies is confined to patients with KRAS wild-type tumors, with no benefit likely to be derived where the tumor carries a KRAS mutation [6-8]. Little evidence is available, however, on which type of tumor is more likely to respond to cetuximab, indicating the urgent need for further study to determine supplementary predictive factors.

Human CD133, a five-transmembrane domain glycoprotein, was initially described as a surface antigen present on human hematopoietic stem cells. Cancer stem cells (CSCs) are a small sub-population of cancer cells that have stem cell-like properties such as self-renewal and the ability to differentiate into multiple cell types, and are referred to as tumor-initiating cells due to these properties [9]. There is now a growing body of evidence to support the hypothesis that CSCs play a major role in cancer progression, possessing the ability for self-renewal, which is believed to drive tumor growth [10]. Previous studies have shown that CD133 is a useful marker for the detection of tumor initiating cells, and CD133 has been implicated in several solid tumor types, including CRC [11-14]. Although initial reports indicated that expression of CD133 was predictive of response to chemotherapy, subsequent studies showed that CD133 expression was irrespective of tumorogenicity. Lugli et al. reported that CD133 status was not significantly associated with tumor progression or survival $[15,16]$. The prognostic significance of CD133 derived from mCRC is still under debate, and further immunohistochemical study of CD133 and comparison of the results with clinicopathological features are necessary.

To our knowledge, no studies to date have investigated the

*Corresponding author: Satoshi Matsusaka, 3-8-31 Ariake, Koto-ku, Tokyo 135-8550, Japan, Tel: +81-3-3520-0111; Fax: +81-3-3570-0343; E-mail: satoshi.matsusaka@jfcr.or.jp

Received November 13, 2013; Accepted December 04, 2013; Published December 06, 2013

Citation: Inoue D, Matsusaka S, Yamamoto N, Suenaga M, Shinozaki E, et al. (2013) CD133-positive Status Predicts Better Prognosis in Metastatic Colorectal Cancer Patients Treated with Cetuximab. J Cytol Histol 5: 202. doi:10.4172/21577099.1000202

Copyright: @ 2013 Inoue D, et al. This is an open-access article distributed under the terms of the Creative Commons Attribution License, which permits unrestricted use, distribution, and reproduction in any medium, provided the original author and source are credited. 
relationship between CD133 status and prognosis in mCRC patients treated with cetuximab. The purpose of this study was to evaluate the prognostic significance of CD133 expression as a biomarker in mCRC patients treated with cetuximab in combination chemotherapy.

\section{Materials and Methods}

\section{Patients}

Fifty-five patients were treated with cetuximab as salvage therapy at the Cancer Institute Hospital of the Japanese Foundation for Cancer Research between October 2008 and June 2009. As a strategy for treatment of $\mathrm{mCRC}$ in our institution, patients received oxaliplatinbased regimen with or without bevacizumab as first-line therapy, followed by irinotecan-based regimen as salvage-line therapy. KRAS wild type patients with $\mathrm{mCRC}$ were also considered to receive cetuximab as salvage-line.

A total of 46 patients with $\mathrm{mCRC}$ were evaluable and included in the current analysis. Selection criteria were as follows: histologically confirmed colorectal adenocarcinoma; Eastern Cooperative Oncology Group (ECOG) performance status of 0 or 1 ; and adequate organ function. Immunohistochemical evidence of KRAS wild-type, either in the primary tumor or in at least one metastatic lesion, was required.

Antitumor effects were evaluated by computed tomography imaging, and were performed at baseline and repeated every 2-3 months till progression.

This study was approved by the Institutional Review Committee of the Cancer Institute Hospital of the Japanese Foundation for Cancer Research.

\section{Immunohistochemistry for CD133}

Immunohistochemistry for CD133 was analyzed using specimens, which were either resected specimens from primary lesion or biopsy specimens before chemotherapy. Status of CD133 after chemotherapy was not evaluated in this study.

Four $\mu \mathrm{m}$-thick specimens of formalin-fixed paraffin-embedded tissue were prepared. More than two different sections were stained per case to confirm reproducibility. After deparaffinization and dehydration, antigen unmasking was accomplished by bringing the specimens to the boil in $10 \mathrm{mM}$ sodium citrate buffer. Specimens were then blocked and incubated with primary antibody overnight at $4^{\circ} \mathrm{C}$. Bound antibody was detected with Envision reagents (Envision kit/ HRP, Dako Cytomation, Denmark). All sections were counterstained with hematoxylin. Primary anti-CD133 rabbit monoclonal antibody (clone C24B9, Cell Signaling Technology, Denvers, MA, USA) was used at a dilution of 1:100 and detection performed by the HRP-labeled streptavidin/biotin method (LASB2 kit/HRP, Dako Cytomation).

For evaluation of immunohistochemistry of CD133, positive staining was defined as luminal surface staining with or without intraluminal content; staining of shed cellular debris was also defined as positive.

\section{KRAS direct sequence}

All tumor tissues were identified to be KRAS wild-type by DNA extraction and direct sequencing using proteinase- $K$ digestion and phenol/chloroform extraction according to the method of Sambrook et al.

\section{Statistical analysis}

Kaplan-Meier survival plots were generated based on CD133 status and the curves compared by using the log-rank test. A Cox proportional hazards regression model was used to determine univariate and multivariate hazard ratios for progression-free survival (PFS) and overall survival (OS) with cetuximab not only as salvage, but also as first-line therapy.

\section{Results}

\section{Patient characteristics}

A total of 46 patients were evaluable. Patient characteristics before administration of cetuximab are summarized in Table 1. As for the status of primary tumor, 11 patients were metastatic cancer at the diagnosis and 35 patients had recurrence after curative resection for primal lesion. All patients received a 5-FU-based regimen such as FOLFOX as a first-line regimen, 17 patients received one regimen and 29 received two regimens prior to cetuximab. As a salvage regimen, 10 patients were treated with cetuximab alone, 21 with cetuximab and irinotecan, and 15 with cetuximab and FOLFIRI.

At the time of analysis, all patients had disease progression for salvage-line chemotherapy, and 45 patients $(97.8 \%)$ died within that time period. Median follow-up period was 29.4 months.

Doses of cetuximab and other chemotherapeutic agents were delayed or reduced if patients had clinically significant adverse events. There were no severe adverse event to cetuximab developed and all the patients received cetuximab till disease progression or unacceptable toxic effects caused by other chemotherapeutic agents occurred.

We studied the association between CD133 status and response and survival in patients with mCRC treated with cetuximab. Response and survival with a 5-FU-based regimen as first-line chemotherapy and cetuximab as salvage-line therapy were analyzed.

\begin{tabular}{|l|c|}
\hline & Number or median \\
\hline CD133: positive/negative & $32 / 14$ \\
\hline Median age (range) & $59.1(28-80)$ \\
\hline Sex: male/female & $29 / 17$ \\
\hline PS: $0 / 1 / 2$ & $34 / 12 / 0$ \\
\hline Primary site: colon/rectum & $30 / 16$ \\
\hline Status of primary tumor: Resected/Unresected & $35 / 11$ \\
\hline Sites of metastasis: + /- & \\
\hline Liver & $38 / 8$ \\
\hline Lung & $33 / 13$ \\
\hline Bone & $6 / 40$ \\
\hline Peritoneum & $8 / 38$ \\
\hline Lymph node & $13 / 33$ \\
\hline Local recurrence & $3 / 43$ \\
\hline Prior treatment: & \\
\hline 5FU & $15 / 21 / 10$ \\
\hline Irinotecan & $23 / 23$ \\
\hline Oxaliplatin & $38 / 8$ \\
\hline Bevacizumab & 46 \\
\hline No. of previous chemotherapy: one/two & 30 \\
\hline Treatment: & 44 \\
\hline FOLFIRI with Cmab/ PT11 with Cmab/Cmab alone & 22 \\
\hline CA19-9: $>37.5 / \leq 37.5$ & $17 / 29$ \\
\hline CEA: $>5 / \leq 5$ & \\
\hline & \\
\hline
\end{tabular}

PS: performance status; Cmab: cetuximab.

Table 1: Baseline characteristics. 


\section{Relationship between CD133 status and response rate}

Tumor response to treatment was classified according to the standard World Health Organization response criteria. In salvage therapy, we observed a complete response in $1(2.3 \%)$, partial response in $3(6.5 \%)$, stable disease in $21(48.8 \%)$, and progressive disease in 21 patients $(48.8 \%)$ during treatment. The overall response rate was $7.8 \%$. The response rate in CD133-positive patients was $9.3 \%$, while that in CD133-negative patients was $7.1 \%$.

In first-line therapy, the overall response rate was $45.7 \%$. The response rate in CD133-positive patients was $46.9 \%$, while that in CD133-negative patients was $42.9 \%$. No significant difference was observed between CD133 status and response rate in either salvage or first-line therapy.
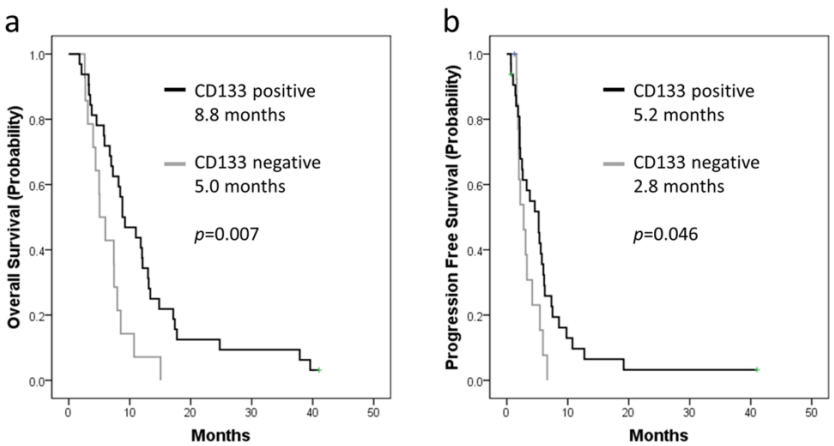

Figure 1: Kaplan-Meier curves for overall survival (a), progression-free survival (b). CD133-positive indicated significantly longer PFS and OS than CD133negative.

\section{Relationship between CD133 status and PFS and OS in first- line chemotherapy}

In first-line chemotherapy, the median OS was 33.1 months (95\% CI, 25.6-40.4) in the CD133-positive patients as compared with 16.0 months (95\% CI, 0.938-31.1) in the CD133-negative patients $(P=0.004)$. However, the median PFS was not statistically significantly better in CD133-positive patients, with a median of 12.0 month (95\% CI, 8.5815.4) in the CD133-positive patients as compared with 5.6 month $(95 \%$ CI, 0.528-10.7) in CD133-negative patients $(P=0.246)$.

In the univariate Cox proportional hazards regression analysis, no parameters were associated with PFS. On the other hand, in the univariate and multivariate Cox proportional hazards regression analysis, CD133-positive was the strongest predictor of OS in first-line chemotherapy (Table 2).

\section{Relationship between CD133 status and PFS and OS in salvage-line therapy with cetuximab}

In salvage therapy with cetuximab, the median OS was 8.8 months (95\% CI, 5.24-12.4) in the CD133-positive patients as compared with 5.1 months (95\% CI, 3.23-6.90) in the CD133-negative patients $(P=0.007)$.

The median PFS was also significantly better in CD133-positive patients, with a median of 5.2 month (95\% CI, 2.96-7.51) in the CD133positive patients as compared with 2.8 month (95\% CI, 1.48-4.06) in CD133-negative patients $(P=0.046)$ (Figure 1).

According to the univariate Cox regression analysis, CD133positive tumor cells tended to indicate a longer PFS (HR 0.500, 95\% CI, 0.249-1.003, $P=0.051$ ), and patients with peritoneal metastasis tended to have a shorter PFS (HR 2.019, 95\% CI, 0.907-4.495, $P=0.085$ ).

\begin{tabular}{|c|c|c|c|c|c|c|}
\hline \multicolumn{7}{|l|}{ Univariate Cox regression analysis } \\
\hline \multirow{2}{*}{ Parameter } & \multicolumn{3}{|c|}{ PFS } & \multicolumn{3}{|c|}{ OS } \\
\hline & HR & $95 \% \mathrm{Cl}$ & p-value & HR & $95 \% \mathrm{Cl}$ & p-value \\
\hline Sex (female) & 1.611 & $0.837-3.099$ & 0.153 & 1.823 & $0.962-3.458$ & 0.066 \\
\hline Age $(>65)$ & 1.019 & $0.540-1.924$ & 0.953 & 1.742 & $0.925-3.282$ & 0.086 \\
\hline PS (1) & 1.006 & $0.484-2.092$ & 0.987 & 1.240 & $0.637-2.414$ & 0.527 \\
\hline CA19-9 (>37.5) & 1.289 & $0.621-2.567$ & 0.451 & 1.287 & $0.599-2.532$ & 0.351 \\
\hline CEA $(>5)$ & 1.311 & $0.654-2.444$ & 0.397 & 1.214 & $0.673-2.479$ & 0.359 \\
\hline Status of primary tumor (resected) & 0.582 & $0.286-1.184$ & 0.135 & 0.537 & $0.266-1.086$ & 0.084 \\
\hline \multicolumn{7}{|l|}{ Metastasis site } \\
\hline Liver & 1.129 & $0.496-2.570$ & 0.773 & 0.852 & $0.394-1.843$ & 0.685 \\
\hline Lung & 0.741 & $0.364-1.413$ & 0.343 & 0.752 & $0.351-1.484$ & 0.363 \\
\hline Bone & 1.033 & $0.432-2.470$ & 0.941 & 1.173 & $0.489-2.815$ & 0.721 \\
\hline Peritoneum & 0.730 & $0.333-1.602$ & 0.433 & 1.061 & $0.491-2.294$ & 0.880 \\
\hline Lymph node & 1.016 & $0.526-1.962$ & 0.963 & 1.126 & $0.585-2.167$ & 0.722 \\
\hline Local recurrence & 0.278 & $0.066-1.171$ & 0.081 & 0.512 & $0.157-1.671$ & 0.268 \\
\hline CD133 positive & 0.682 & $0.355-1.309$ & 0.250 & 0.391 & $0.203-0.756$ & 0.005 \\
\hline \multicolumn{7}{|l|}{ Multivariate Cox regression analysis } \\
\hline \multirow{2}{*}{ Parameter } & \multicolumn{3}{|c|}{ PFS } & \multicolumn{3}{|c|}{ OS } \\
\hline & HR & $95 \% \mathrm{Cl}$ & p-value & HR & $95 \% \mathrm{Cl}$ & p-value \\
\hline Sex (female) & 1.839 & $0.889-3.806$ & 0.101 & 1.941 & $0.953-3.551$ & 0.051 \\
\hline Age $(>65)$ & 1.581 & $0.763-3.277$ & 0.218 & 2.842 & $1.273-6.342$ & 0.011 \\
\hline Status of primary tumor (resected) & 0.567 & $0.266-1.209$ & 0.142 & 0.309 & $0.137-0.698$ & 0.005 \\
\hline Local recurrence & 0.314 & $0.066-1.488$ & 0.144 & 0.641 & $0.162-2.537$ & 0.526 \\
\hline CD133 positive & 0.759 & $0.387-1.490$ & 0.423 & 0.339 & $0.162-0.711$ & 0.004 \\
\hline
\end{tabular}

PFS: Progression-Free Survival; OS: Overall Survival; HR: Hazard Ratio; PS: Performance Status; Mg: Magnesium.

Table 2: Univariate and multivariate Cox proportional hazards regression in first-line chemotherapy. 
Citation: Inoue D, Matsusaka S, Yamamoto N, Suenaga M, Shinozaki E, et al. (2013) CD133-positive Status Predicts Better Prognosis in Metastatic Colorectal Cancer Patients Treated with Cetuximab. J Cytol Histol 5: 202. doi:10.4172/2157-7099.1000202

Page 4 of 6

In terms of OS, univariate Cox regression analysis showed that CD133-positive (HR 0.405, 95\% CI, 0.205-0.798, $P=0.009$ ) and peritoneal metastasis (HR 2.879, 95\% CI, 1.266-6.549, $P=0.008$ ) were predictive of OS. A multivariate Cox regression analysis was carried out to evaluate the independent predictive effect of these markers, with the
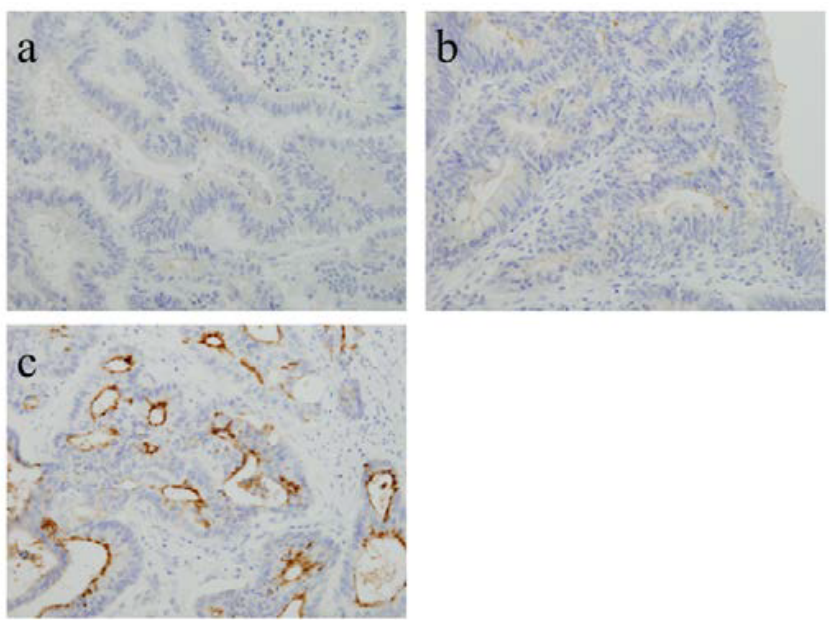

Figure 2: Intensity of staining was scored on scale of 0 to 2 , in which 0 indicated negative (a), 1 weakly positive (b), and 2 strongly positive staining (c). Positive staining was defined as luminal surface staining with or without intraluminal content. Original magnification $200 \times(a)$ and $100 \times(b$ and $c)$. results revealing that $\mathrm{CD} 133$ status was the strongest predictor of OS (Table 3).

\section{Relationship between intensity of immunohistochemistry for CD133 and prognosis}

As stated above, CD133-positive tumors indicated significantly longer survival in salvage-line chemotherapy with cetuximab. The relationship between tumor CD133 expression level and outcome was also evaluated.

The intensity of staining was scored on a scale of 0 to 2 , in which 0 indicated negative, 1 weakly positive, and 2 strongly positive staining (Figure 2).

Ten of the specimens were scored as 2 and 22 as 1 . No statistically significant difference was observed in OS or PFS between the 0 score and 1-2 group. On the other hand, no significant difference was observed between the $0-1$ and 2 groups, suggesting that intensity of staining was not an indicator of prognosis in first- or salvage-line therapy.

\section{Discussion}

The results of the present study showed that positive expression of CD133 was associated with better response to cetuximab, with a significantly longer OS and PFS in salvage-line therapy. To our knowledge, this is the first study to analyze the relationship between CD133 and prognosis in mCRC patients treated with cetuximab.

Some reports have indicated that CD133 status influenced

Univariate Cox regression analysis

\begin{tabular}{|c|c|c|c|c|c|c|}
\hline \multirow{2}{*}{ Parameter } & \multicolumn{3}{|c|}{ PFS } & \multicolumn{3}{|c|}{ os } \\
\hline & HR & $95 \% \mathrm{Cl}$ & p-value & HR & $95 \% \mathrm{Cl}$ & p-value \\
\hline Sex (female) & 1.504 & $0.807-2.802$ & 0.198 & 1.396 & $0.758-2.571$ & 0.285 \\
\hline Age $(>65)$ & 1.317 & $0.693-2.502$ & 0.401 & 1.813 & $0.950-3.460$ & 0.071 \\
\hline PS (1) & 1.500 & $0.763-2.948$ & 0.240 & 1.41 & $0.714-2.787$ & 0.322 \\
\hline CA19-9 (>37.5) & 1.388 & $0.712-2.403$ & 0.399 & 1.382 & $0.746-2.478$ & 0.389 \\
\hline $\operatorname{CEA}(>5)$ & 1.369 & $0.698-2.488$ & 0.411 & 1.397 & $0.765-2.494$ & 0.412 \\
\hline Status of primary tumor (resected) & 0.972 & $0.487-1.938$ & 0.935 & 0.742 & $0.373-1.479$ & 0.397 \\
\hline \multicolumn{7}{|l|}{ Metastasis site } \\
\hline Liver & 0.542 & $0.246-1.198$ & 0.130 & 0.593 & $0.271-1.297$ & 0.191 \\
\hline Lung & 0.585 & $0.288-1.187$ & 0.137 & 0.74 & $0.384-1.426$ & 0.369 \\
\hline Bone & 1.549 & $0.640-3.752$ & 0.332 & 1.776 & $0.720-4.381$ & 0.212 \\
\hline Peritoneum & 2.019 & $0.907-4.495$ & 0.085 & 2.879 & $1.266-6.549$ & 0.012 \\
\hline Lymph node & 1.601 & $0.801-3.202$ & 0.183 & 1.629 & $0.844-3.147$ & 0.146 \\
\hline Local recurrence & 0.832 & $0.255-2.720$ & 0.761 & 0.996 & $0.306-3.249$ & 0.995 \\
\hline CD133 positive & 0.500 & $0.249-1.003$ & 0.051 & 0.405 & $0.205-0.798$ & 0.009 \\
\hline \multicolumn{7}{|l|}{ Multivariate Cox regression analysis } \\
\hline \multirow{2}{*}{ Parameter } & \multicolumn{3}{|c|}{ PFS } & \multicolumn{3}{|c|}{ os } \\
\hline & HR & $95 \% \mathrm{Cl}$ & $p$-value & HR & $95 \% \mathrm{Cl}$ & p-value \\
\hline Sex (female) & 1.456 & $0.712-2.977$ & 0.303 & 1.457 & $0.721-2.467$ & 0.152 \\
\hline Age $(>65)$ & 2.666 & $1.063-6.684$ & 0.037 & 2.442 & $1.082-5.510$ & 0.032 \\
\hline PS (1) & 1.693 & $0.814-3.521$ & 0.159 & & & \\
\hline Liver & 0.732 & $0.270-1.986$ & 0.541 & 1.251 & $0.511-3.067$ & 0.624 \\
\hline Lung & 0.867 & $0.374-2.008$ & 0.739 & & & \\
\hline Bone & & & & 1.008 & $0.371-2.735$ & 0.988 \\
\hline Peritoneum & 1.958 & $0.768-4.993$ & 0.160 & 1.772 & $0.669-4.695$ & 0.250 \\
\hline Lymph node & 1.194 & $0.546-2.610$ & 0.657 & 0.755 & $0.355-1.606$ & 0.466 \\
\hline CD133 positive & 0.559 & $0.259-1.209$ & 0.140 & 0.316 & $0.146-0.686$ & 0.004 \\
\hline
\end{tabular}

Table 3: Univariate and multivariate Cox proportional hazards regression in salvage-line therapy. 
prognosis in patients with mCRC, with most of them showing that CD133-positive was a predictor of poor survival and suggesting that CD133 might be a CSC marker in mCRC [11-14]. Ong et al. performed immunohistochemistry for CD133 and other prognostic markers, and multivariate analysis revealed that positive expression for CD133 was associated with significantly worse OS in patients treated with 5 -FUbased chemotherapy [17].

The results of the present study are in disagreement with those of these earlier investigations. In this study, CD133-positive indicated a significantly longer OS, but not significantly better PFS, in a first-line 5-FU-based regimen. We believe that the longer OS observed here may have resulted from the longer OS achieved in salvage-line therapy. This suggests that CD133-positive does not predict prognosis in a 5-FUbased regimen.

\section{Criteria and definition of immunohistochemistry for CD133}

In earlier studies, the ratio of CD133-positive tumors was reported to range from $15.3 \%$ to $26.0 \%$, and the criteria for and definition of a CD133-positive tumor were different [18-20].

Horst et al. analyzed surgically treated CRC patients and showed that CD133 over-expression was a risk factor for poorer OS. In that study, a gland was considered positive if either membranous staining or staining of shed cellular debris was observed. Moreover, high expression of CD133 was defined as a tumor showing more than $50 \%$ positive glands. According to these criteria, $26 \%$ of the tumors were CD133-high [13].

Kojima et al. also found that CD133-positive cases had a poorer outcome than CD133-negative ones in surgically treated colorectal cancer patients. In their study, positive expression of CD133 was only observed on the cell membrane at the liminal surface of the glands, with CD133 expression being detected in $15.3 \%$. They defined CD133positive cases as where more than $10 \%$ of the glands were positive in the cancer-affected area [18].

Saigusa et al. examined patients with rectal cancer who had received preoperative chemoradiotherapy, and referred to cytoplasmic CD133 expression. They concluded that the ratio of histopathological responders in cases with CD133 expression in both the luminal surface and cytoplasm was low [19].

In the present study, for evaluation of immunohistochemistry for CD133, we defined positive staining as luminal surface staining with or without intraluminal content, and we did not take cytoplasmic expression of CD133 into account. The ratio of CD133-positive tumors was $70.0 \%$, which was higher than that in other reports. This was because, here, CD133-positive was defined according to the intensity of the CD133 stain only, not to the range of positive cells in canceraffected areas. Even if less than $10 \%$ of the cells had been CD133positive in the cancer-affected area, the tumor would still have been defined as CD133-positive according to the criteria of the present study.

One problem with CD133 immunohistochemistry is that the criteria for CD133-positive remain to be established.

\section{EGFR inhibitor and CD133 expression}

In breast cancer, Chang et al. showed that lapatinib (Tykerb), an EGFR/HER2 inhibitor, can cause complete tumor regression, possibly by killing CD44+ and CD24- cells. They examined paired breast cancer biopsies before and after treatment with lapatinib for HER2-positive tumors. The results showed that lapatinib treatment led to a non- statistically significant decrease in the percentage of CD44+ and CD24or low cells, whereas chemotherapy led to an increase [20].

The biological function of CD133-positive cells, and also CD44+/ CD24- cells, is not yet well understood, and the reason these tumors show a better response to EGFR inhibitors is unclear. However, the existence of a subpopulation which has a better response to EGFR inhibitors such as lapatinib and cetuximab is suggested.

This study has several potential limitations, one of which was the relatively small sample size. The functional significance of CD133+ cells is unclear. Therefore, their importance in metastasis, disease recurrence, and CRC mortality is not yet well understood.

In summary, there was possibility that positive expression of CSC marker CD133 was associated with better survival in mCRC patients treated with cetuximab.

\section{Acknowledgements}

The authors would like to thank Ms. Tomoyo Kakita and Mr. Genkichi Iwakosh for their kind advisory and technical assistance at the department of Pathology Cancer Institute Hospital of the Japanese Foundation for Cancer Research.

\section{References}

1. Saltz LB, Meropol NJ, Loehrer PJ Sr, Needle MN, Kopit J, et al. (2004) Phase II trial of cetuximab in patients with refractory colorectal cancer that expresses the epidermal growth factor receptor. J Clin Oncol 22: 1201-1208.

2. Lenz HJ, Van Cutsem E, Khambata-Ford S, Mayer RJ, Gold P, et al. (2006) Multicenter phase II and translational study of cetuximab in metastatic colorectal carcinoma refractory to irinotecan, oxaliplatin, and fluoropyrimidines. $\mathrm{J}$ Clin Oncol 24: 4914-4921.

3. Tabernero J, Van Cutsem E, Díaz-Rubio E, Cervantes A, Humblet $Y$, et al (2007) Phase II trial of cetuximab in combination with fluorouracil, leucovorin and oxaliplatin in the first-line treatment of metastatic colorectal cancer. J Clin Oncol 25: 5225-5232.

4. Arnold D, Höhler T, Dittrich C, Lordick F, Seufferlein T, et al. (2008) Cetuximab in combination with weekly 5 -fluorouracil/folinic acid and oxaliplatin (FUFOX) in untreated patients with advanced colorectal cancer: a phase Ib/ll study of the AIO GI Group. Ann Oncol 19: 1442-1449.

5. Van Cutsem E, Köhne CH, Hitre E, Zaluski J, Chang Chien CR, et al. (2009) Cetuximab and chemotherapy as initial treatment for metastatic colorectal cancer. N Engl J Med 360: 1408-1417.

6. Lièvre A, Bachet JB, Boige V, Cayre A, Le Corre D, et al. (2008) KRAS mutations as an independent prognostic factor in patients with advanced colorectal cancer treated with cetuximab. J Clin Oncol 26: 374-379.

7. Yen LC, Uen YH, Wu DC, Lu CY, Yu FJ, et al. (2010) Activating KRAS mutations and overexpression of epidermal growth factor receptor as independent predictors in metastatic colorectal cancer patients treated with cetuximab. Ann Surg 251: 254-260

8. Allegra CJ, Jessup JM, Somerfield MR, Hamilton SR, Hammond EH, et al (2009) American Society of Clinical Oncology provisional clinical opinion: testing for KRAS gene mutations in patients with metastatic colorectal carcinoma to predict response to anti-epidermal growth factor receptor monoclonal antibody therapy. J Clin Oncol 27: 2091-2096.

9. Boman BM, Huang E (2008) Human colon cancer stem cells: a new paradigm in gastrointestinal oncology. J Clin Oncol 26: 2828-2838.

10. Reya T, Morrison SJ, Clarke MF, Weissman IL (2001) Stem cells, cancer, and cancer stem cells. Nature 414: 105-111.

11. Horst D, Kriegl L, Engel J, Kirchner T, Jung A (2009) Prognostic significance of the cancer stem cell markers CD133, CD44, and CD166 in colorectal cancer Cancer Invest 27: 844-850

12. O'Brien CA, Pollett A, Gallinger S, Dick JE (2007) A human colon cancer cell capable of initiating tumour growth in immunodeficient mice. Nature 445: 106 110.

13. Ricci-Vitiani L, Lombardi DG, Pilozzi E, Biffoni M, Todaro M, et al. (2007) Identification and expansion of human colon-cancer-initiating cells. Nature 445 111-115. 
Citation: Inoue D, Matsusaka S, Yamamoto N, Suenaga M, Shinozaki E, et al. (2013) CD133-positive Status Predicts Better Prognosis in Metastatic Colorectal Cancer Patients Treated with Cetuximab. J Cytol Histol 5: 202. doi:10.4172/2157-7099.1000202

Page 6 of 6

14. Horst D, Kriegl L, Engel J, Kirchner T, Jung A (2008) CD133 expression is an independent prognostic marker for low survival in colorectal cancer. $\mathrm{Br} J$ Cancer 99: 1285-1289.

15. Shmelkov SV, Butler JM, Hooper AT, Hormigo A, Kushner J, et al. (2008) CD133 expression is not restricted to stem cells, and both CD133+ and CD133metastatic colon cancer cells initiate tumors. J Clin Invest 118: 2111-2120.

16. Lugli A, lezzi G, Hostettler I, Muraro MG, Mele V, et al. (2010) Prognostic impact of the expression of putative cancer stem cell markers CD133, CD166, CD44s, EpCAM, and ALDH1 in colorectal cancer. Br J Cancer 103: 382-390.

17. Ong CW, Kim LG, Kong HH, Low LY, lacopetta B, et al. (2010) CD133 expression predicts for non-response to chemotherapy in colorectal cancer Mod Pathol 23: 450-457.

18. Kojima M, Ishii G, Atsumi N, Fujii S, Saito N, et al. (2008) Immunohistochemical detection of CD133 expression in colorectal cancer: a clinicopathological study. Cancer Sci 99: 1578-1583.

19. Saigusa S, Tanaka K, Toiyama Y, Yokoe T, Okugawa Y, et al. (2010) Immunohistochemical features of CD133 expression: association with resistance to chemoradiotherapy in rectal cancer. Oncol Rep 24: 345-350.

20. Li X, Lewis MT, Huang J, Gutierrez C, Osborne CK, et al. (2008) Intrinsic resistance of tumorigenic breast cancer cells to chemotherapy. J Natl Cancer Inst 100: 672-679. 